\title{
ASSISTÊNCIA À SAÚDE DE PESSOAS QUE FAZEM USO ABUSIVO DE DROGAS EM ESTRATÉGIAS SAÚDE DA FAMÍLIA: REVISÃO DE LITERATURA ${ }^{1}$
}

\author{
HEALTH CARE FOR PEOPLE WHO MAKE DRUG ABUSE IN \\ FAMILY HEALTH STRATEGIES: LITERATURE REVIEW
}

\author{
Júlia Oliveira Silveira ${ }^{2}$, Luane de Oliveira Sena ${ }^{3}$, Naiana Oliveira dos Santos ${ }^{4}$, \\ Zaira Letícia Tisott ${ }^{5}$, Mara Regina Caino Teixeira Marchiori ${ }^{6}$ e Keity Laís Siepmann Soccol ${ }^{7}$
}

\section{RESUMO}

Este estudo teve como objetivo identificar o que vem sendo produzido sobre a assistência à saúde das pessoas que fazem uso abusivo de drogas na Estratégia de Saúde da Família. Trata-se de uma revisão de literatura realizada nas bases de dados Literatura Latino-Americana e do Caribe em Ciências da Saúde (LILACS). A busca ocorreu no mês de setembro de 2020, para as estratégias de busca se utilizou as palavras "usuários de drogas" e "estratégia saúde da família". Foram selecionados para essa revisão dez artigos científicos. Os resultados evidenciam a necessidade de os profissionais identificar e realizar intervenções de cuidado pautadas em ações livres de estigmas com foco na promoção do acolhimento e na escuta. É necessário implicação e capacitação dos profissionais de saúde no cuidado às pessoas que fazem uso abusivo de drogas. Conclui-se, que a atenção à saúde dessas pessoas na Estratégia Saúde da Família deve ser potencializada por meio da criação de políticas públicas e cuidado implicado.

Palavras-chave: Enfermagem, Estratégia Saúde da Família, Saúde Mental, Transtornos Relacionados ao Uso de Substâncias.

\section{ABSTRACT}

This study aimed to identify what has been produced about the health care of people who abuse drugs in the Family Health Strategy. This is a literature review carried out in the Latin American and Caribbean Health Sciences (LILACS) databases. The search took place in September 2020, for the search strategies the words "drug users" and "family health strategy" were used. Ten scientific articles were selected for this review. The results show the need for professionals to identify and carry out care interventions based on actions free

1 Trabalho de iniciação científica relacionado à bolsa de pesquisa do Programa Institucional de Bolsas de Iniciação Científica

2 Autora. Acadêmica do Curso de Graduação em Enfermagem da Universidade Franciscana (UFN). Bolsista do projeto PROBIC. E-mail: oliveirasilveiraj2@outlook.com

3 Autora. Acadêmica do Curso de Graduação em Enfermagem da Universidade Franciscana (UFN). E-mail: senaluane@, gmail.com

4 Autora. Enfermeira. Docente do Curso de Graduação em Enfermagem da Universidade Franciscana (UFN). E-mail: naiaoliveira07@gmail.com

5 Autora. Enfermeira. Mestre em enfermagem. Doutoranda em enfermagem pela Universidade Federal do Rio Grande do Sul (UFRGS). E-mail: zairatisott10@gmail.com

6 Co-orientadora. Enfermeira. Docente do Curso de Graduação em Enfermagem da Universidade Franciscana (UFN). E-mail:mara.marc@hotmail.com

7 Orientadora. Enfermeira. Docente do Curso de Graduação em Enfermagem da Universidade Franciscana (UFN). E-mail: keitylais@hotmail.com 
from stigmas with a focus on promoting welcoming and listening. It is necessary to involve and train health professionals in caring for people who abuse drugs. It is concluded that the health care of these people in the Family Health Strategy should be enhanced through the creation of public policies and implied care.

Keywords: Nursing, Family health strategy, Mental health, Substance-related disorders.

\section{INTRODUÇÃO}

A Reforma Psiquiátrica no Brasil foi idealizada internacionalmente por movimentos sociais que visavam mudanças no regime manicomial orientada pelo fim da violência asilar. O processo contou com o empenho de diversos atores sociais, que acreditavam ser possível um novo modelo assistencial em saúde mental. A partir da Lei nº 10.216/2001 a Rede de Atenção Psicossocial (RAPS) toma forma, onde a base comunitária de cuidado é o foco da atenção. Dentre as propostas substitutivas ao modelo manicomial, destacam-se aqueles em âmbito territorial, como os Centros de Atenção Psicossocial (CAPS), as Unidades Básicas de Saúde (UBS) e as Estratégias Saúde da Família (ESF) (BRASIL, 2005).

A Estratégia Saúde da Família (ESF) tem como propósito a prestação de cuidados à saúde dos indivíduos e famílias do território, por meio do vínculo contínuo entre equipe e comunidade, onde todas as ações são planejadas de acordo com a realidade da população que se pretende atender. Levando em consideração os determinantes sociais de saúde e doença em cada um dos contextos, de forma integral e resolutiva. O uso habitual de drogas é um exemplo de determinante que interfere no bem-estar individual e social (LIRA et al., 2020).

A ESF constitui-se como uma ferramenta de cuidado que envolve a promoção da saúde frente a realidade social da população adscrita, e dessa maneira, contribui significativamente sobre ela (PNAB, 2017). Ainda, é um dos dispositivos que mais atendem as pessoas que fazem uso abusivo de drogas, pois esta está próxima de onde elas moram, ou seja, está inserida no território (SINIAK et al., 2021). Desse modo, o enfermeiro enquanto gestor da ESF pode ser considerado um profissional estratégico para fortalecer a atenção primária enquanto lócus de cuidado em saúde mental e articuladora da rede intersetorial (ESLABÃO et al., 2017).

O uso drogas é considerado um problema de saúde pública, sobretudo quando os serviços de saúde não logram êxito no combate ao uso desenfreado, cada vez mais cedo, por adolescentes e jovens, e de substâncias cada vez mais nocivas, como o crack. Além disso, o estigma socialmente construído de que a pessoa que faz uso abusivo de drogas é marginalizada, em situação de rua, violenta, e como vetor de Infeções Sexualmente Transmissíveis (IST’s), entre outros, colabora para o afastamento delas dos meios de cuidado em saúde (FARIAS et al., 2017). A atenção básica se configura como um local para a ampliação de cuidado às pessoas que consomem bebidas alcoólicas e outras drogas, a qual 
tende a deslocar a imagem de cuidado em saúde mental vinculado apenas aos centros especializados (SANTOS; FERLA, 2017).

Nessa perspectiva, o conhecimento acerca das práticas e condutas dos profissionais que atuam na ESF é fundamental para a avaliação da efetividade das políticas públicas, bem como das ações e serviços da RAPS. Tendo em vista que estes profissionais têm a responsabilidade sanitária pelo território de adscrição da ESF, são capazes de realizar o rastreamento, a busca ativa, o acompanhamento multiprofissional e a redução dos danos do uso abusivo de drogas (FARIAS et al., 2019).

Haja visto a importância desse dispositivo na RAPS e sobre o papel dos profissionais de saúde para o desenvolvimento do cuidado, esse estudo objetiva identificar o que vem sendo produzido sobre a assistência à saúde das pessoas que fazem uso abusivo de drogas na Estratégia de Saúde da Família.

\section{METODOLOGIA}

Trata-se de um estudo do tipo revisão narrativa de literatura. Esse tipo de estudo permite identificar a produção do conhecimento acerca de um determinado assunto/tema. As revisões podem ser realizadas por meio da análise de artigos, livros entre outros documentos. E, a interpretação desses dados permite uma análise crítica (SALLUM et al., 2012).

Essa revisão foi realizada por meio de um levantamento de produções científicas que constavam na Biblioteca eletrônica Scientific Electronic Library Online (SCIELO) e na Literatura Latino-americana e do Caribe em Ciências da Saúde (LILACS). A questão norteadora desse estudo foi: qual a tendência da produção científica brasileira sobre a assistência à saúde das pessoas que fazem uso abusivo de drogas na Estratégia Saúde da Família?

Para o levantamento das produções científicas utilizou-se as seguintes palavras em ambas as bases de dados: "usuários de drogas" e "estratégia saúde da família”. Ainda, refinou-se a busca com a escolha do país Brasil, na qual se utilizou somente estudos desenvolvidos nesse país. Não se utilizou seleção de idiomas e nem de recorte temporal para abranger o maior número de estudos possíveis. A busca foi realizada no mês de setembro do ano de 2020.

Como critérios de inclusão tiveram-se: os artigos originais que respondessem ao objetivo proposto, online, gratuito, na íntegra e desenvolvido no Brasil. E, como critérios de exclusão: artigos de revisão, teses, dissertações, resumos de conferências e manuais ministeriais. A busca na SCIELO resultou em 15 artigos. Após a leitura foram excluídos seis artigos pois os mesmos não respondiam ao objetivo proposto, assim selecionou-se nove artigos nessa base de dados. E, na LILACS resultou em dois, no qual se utilizou um artigo, pois o outro estava repetido nas duas bases de dados. Após aplicação dos critérios de inclusão e exclusão utilizou-se um total de 10 artigos, que foram lidos e analisados na íntegra. 
Figura 1

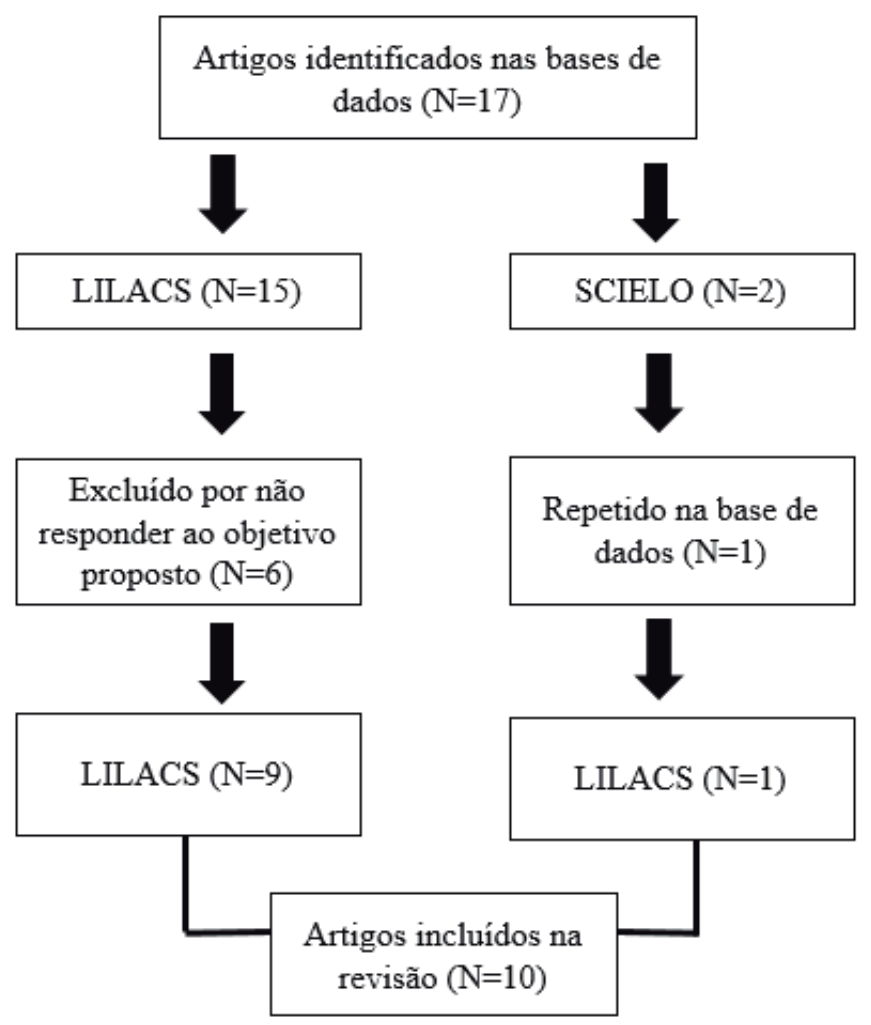

Fonte: construção dos autores.

\section{RESULTADOS E DISCUSSÕES}

A amostra final desta revisão constituiu-se de 10 artigos relacionados ao foco deste estudo. Para a apresentação dos resultados elaborou-se um quadro sinóptico, no qual os dados foram organizados. Esse quadro contém o código de identificação, que corresponde à ordem em que o estudo foi citado no texto, a referência do artigo, o objetivo e os principais resultados. Os artigos que constituíram o corpus de análise estão apresentados no Quadro 1.

Quadro 1 - Artigos que compuseram o corpus de análise

\begin{tabular}{|c|c|c|c|}
\hline $\begin{array}{c}\text { Código de identificação/ } \\
\text { Referência }\end{array}$ & $\begin{array}{c}\text { Base de } \\
\text { dados }\end{array}$ & Objetivo & Principais resultados \\
\hline $\begin{array}{l}\text { A1 - Abreu, A.M.M.; Jomar, R.T.; } \\
\text { Taets, G.G.C.; Souza, M.H.N. ; } \\
\text { Fernandes, D.B.; Rastreamento e } \\
\text { Intervenção Breve para o uso de } \\
\text { álcool e outras drogas. Rev Bras } \\
\text { Enferm. v. 71, n. 5, p. 2389-95, } 2018 .\end{array}$ & SCIELO & $\begin{array}{l}\text { Identificar o uso, na vida, de álcool } \\
\text { e outras drogas entre usuários } \\
\text { da Estratégia Saúde da Família e } \\
\text { aplicar intervenção breve para } \\
\text { problemas relacionados ao uso } \\
\text { dessas substâncias. }\end{array}$ & $\begin{array}{l}\text { As drogas mais usadas na vida foram } \\
\text { álcool e tabaco; entre as drogas } \\
\text { ilícitas, destacaram-se maconha, } \\
\text { hipnóticos e cocaína/crack. Os que } \\
\text { mais receberam intervenção breve } \\
\text { foram os usuários de tabaco, hipnóti- } \\
\text { cos, maconha, cocaína/crack e álcool. }\end{array}$ \\
\hline
\end{tabular}




\begin{tabular}{|c|c|c|c|}
\hline $\begin{array}{l}\text { A2 - Coelho, H.V. ; Soares, C.B.; } \\
\text { Práticas na Atenção Básica voltadas } \\
\text { para o consumo prejudicial de } \\
\text { drogas. Rev Esc Enferm USP, } \\
\text { v. 48, n. 9, p. 111-118, } 2014 .\end{array}$ & SCIELO & $\begin{array}{l}\text { Analisar as práticas de atenção } \\
\text { básica voltadas ao consumo } \\
\text { prejudicial de drogas. }\end{array}$ & $\begin{array}{l}\text { As demandas não são acolhidas e, } \\
\text { quando ultrapassam as barreiras, } \\
\text { não são reinterpretadas como ne- } \\
\text { cessidades de saúde; as práticas que } \\
\text { atendem essas demandas são precá- } \\
\text { rias; a perspectiva funcionalista, que } \\
\text { compreende o consumo de drogas } \\
\text { como doença e considera usuários } \\
\text { de drogas como desviantes embasam } \\
\text { as escassas práticas existentes. }\end{array}$ \\
\hline $\begin{array}{l}\text { A3 - Farias, L.; Bernardino, I.M.; } \\
\text { Madruga, R.C.R.; D’avila, S.; } \\
\text { Lucas, R.S.C.C.; Atitudes e práticas } \\
\text { de profissionais atuantes na } \\
\text { Estratégia Saúde da Família quanto } \\
\text { à abordagem aos usuários de drogas } \\
\text { no município de Campina Grande, } \\
\text { Paraíba, Brasil. Ciência \& Saúde } \\
\text { Coletiva, v. } 24, \text { n. } 10, \text { p. } 3867-3878, \\
2019 .\end{array}$ & SCIELO & $\begin{array}{l}\text { Avaliar as atitudes e as práticas dos } \\
\text { profissionais da Estratégia Saúde da } \\
\text { Família (ESF) quanto à abordagem } \\
\text { aos usuários drogas no município } \\
\text { de Campina Grande, Paraíba. }\end{array}$ & $\begin{array}{l}\text { Quase a totalidade dos participan- } \\
\text { tes relatou conhecer apenas alguns } \\
\text { dos usuários de drogas em sua área } \\
\text { de abrangência, bem como ques- } \\
\text { tionar apenas às vezes quanto ao } \\
\text { uso de drogas durante a anamnese, } \\
\text { Além disso, diante de situações de } \\
\text { urgência, } 37,3 \% \text { responderam que } \\
\text { não saberiam abordar clinicamen- } \\
\text { te e } 22,2 \% \text { destacaram que não } \\
\text { saberiam como e nem para onde } \\
\text { encaminhar o atendimento. }\end{array}$ \\
\hline $\begin{array}{l}\text { A4 - Lira, L.S.S.P. ; Andrade, L.M.; } \\
\text { Peixoto, L.C.P. ; Malhado, S.C.B.; } \\
\text { Sena, E.L.S.; Percepção da Equipe } \\
\text { de Saúde da Família sobre o cuidado } \\
\text { a usuários de drogas. Saúde Debate, } \\
\text { v. } 44 \text {, n. } 125 \text {, p. } 372-383,2020 \text {. }\end{array}$ & SCIELO & $\begin{array}{l}\text { Compreender a percepção da } \\
\text { equipe da Estratégia Saúde da } \\
\text { Família sobre o cuidado a usuários } \\
\text { de drogas. }\end{array}$ & $\begin{array}{l}\text { Olhar o usuário como quem olha } \\
\text { de fora, sem se entrelaçar com ele, } \\
\text { contribui para a segregação e a } \\
\text { exclusão social, o que requer dos } \\
\text { profissionais a suspensão de teses } \\
\text { que sustentam no campo do uso de } \\
\text { drogas e a ressignificação da forma } \\
\text { como veem e cuidam do usuário de } \\
\text { drogas e sua família. }\end{array}$ \\
\hline $\begin{array}{l}\text { A5 - Paula, M.L.; Jorge, M.S.B.; } \\
\text { Vasconcelos, M.G.F.; Albuquerque, } \\
\text { R.A.; Assistência ao Usuário de } \\
\text { Drogas na Atenção Primária à } \\
\text { Saúde. Psicologia em Estudo, } \\
\text { Maringá; v. 19, n. 2, p. 223-233, } \\
\text { abr./jun. 2014. }\end{array}$ & SCIELO & $\begin{array}{l}\text { Analisar a assistência a usuários } \\
\text { de drogas na Atenção Primária à } \\
\text { Saúde a partir dos discursos de } \\
\text { profissionais da ESF. }\end{array}$ & $\begin{array}{l}\text { A APS enfrenta grandes desafios } \\
\text { que fragilizam o cuidado, entre } \\
\text { eles: dificuldade de estabelecimen- } \\
\text { to de vínculo e preconceito contra } \\
\text { os usuários de drogas. As princi- } \\
\text { pais ações estão voltadas à escuta, } \\
\text { ao acolhimento das demandas e ao } \\
\text { aconselhamento às famílias. }\end{array}$ \\
\hline $\begin{array}{l}\text { A6 - Silva, W.R.; Peres, R.S.; } \\
\text { Concepções Sobre Álcool e Outras } \\
\text { Drogas na Atenção Básica: o Pacto } \\
\text { Denegativo dos Profissionais de Saú- } \\
\text { de. Psicologia: Ciência e Profissão; v. } \\
\text { 34, n. 2, p. 474-487, } 2014 .\end{array}$ & SCIELO & $\begin{array}{l}\text { Analisar as concepções de } \\
\text { profissionais de saúde de nível } \\
\text { superior que atuam na Estratégia } \\
\text { de Saúde da Família acerca da } \\
\text { atenção em saúde a usuários de } \\
\text { álcool e outras drogas. }\end{array}$ & $\begin{array}{l}\text { Os participantes possuem concep- } \\
\text { ções acerca dos usuários permea- } \\
\text { das por preconceitos e estigmas, o } \\
\text { que gera dificuldades para o em- } \\
\text { preendimento de ações de saúde e } \\
\text { os leva, na maior parte das vezes, a } \\
\text { apenas realizar encaminhamentos. }\end{array}$ \\
\hline
\end{tabular}




\begin{tabular}{|c|c|c|c|}
\hline $\begin{array}{l}\text { A7- Subrinho, L.Q.; Sena, E.L.S.; } \\
\text { Santos, V. T.C.; Carvalho, P. A.L.; } \\
\text { Cuidado ao consumidor de } \\
\text { drogas: percepção de enfermeiros da } \\
\text { Estratégia de Saúde da Família. São } \\
\text { Paulo, v. 27, n. 3, p. 834-844, 2018. } \\
\text { Saúde Soc. }\end{array}$ & SCIELO & $\begin{array}{l}\text { Compreender como enfermeiros } \\
\text { da Estratégia de Saúde da Família } \\
\text { percebem o cuidado aos consu- } \\
\text { midores de drogas nas Unidades } \\
\text { Básicas de Saúde. }\end{array}$ & $\begin{array}{l}\text { Apesar de os enfermeiros reconhece- } \\
\text { rem a necessidade de prestar um cui- } \\
\text { dado integral aos consumidores de } \\
\text { drogas, eles desenvolvem uma assis- } \\
\text { tência que tem como foco prioritário } \\
\text { a abstinência e ainda, culpabilizam } \\
\text { esses consumidores pelo insucesso } \\
\text { no tratamento, ao considerá-los não } \\
\text { responsivos ao cuidado. }\end{array}$ \\
\hline $\begin{array}{l}\text { A8- Schneider, J.F.; Roos, C.M.; } \\
\text { Olschowsky, A.; Pinho, L.B.; } \\
\text { Camatta, M.W.; Wetzel, C.; } \\
\text { Atendimento a Usuários de Drogas } \\
\text { na Perspectiva dos Profissionais da } \\
\text { Estratégia Saúde da Família. Texto } \\
\text { Contexto Enferm, Florianópolis, } \\
\text { v. 22, n. 3, p. 654-61, } 2013 .\end{array}$ & $\mathrm{O}$ & $\begin{array}{l}\text { Avaliar o atendimento a usuários } \\
\text { de drogas no contexto da rede } \\
\text { de atenção em saúde mental a } \\
\text { partir de uma Estratégia Saúde } \\
\text { da Família no município de Porto } \\
\text { Alegre - RS. }\end{array}$ & $\begin{array}{l}\text { Desconexões do serviço com } \\
\text { outros pontos de atenção de saúde } \\
\text { mental do município estudado, } \\
\text { além da existência de precariedade } \\
\text { de estratégias voltadas ao atendi- } \\
\text { mento dos usuários de drogas. }\end{array}$ \\
\hline $\begin{array}{l}\text { A9- Kanno, L.P. ; Bellodi, P. L.; } \\
\text { Tess, B.H.; Profissionais da } \\
\text { Estratégia Saúde da Família diante } \\
\text { de Demandas Médico-Sociais: } \\
\text { dificuldades e estratégias de } \\
\text { enfrentamento. Saúde Soc. } \\
\text { São Paulo, v. 21, n. 4, p. 884-894, } \\
2012 .\end{array}$ & $\mathrm{CO}$ & $\begin{array}{l}\text { Investigar as dificuldades e as } \\
\text { formas de enfrentamento referidas } \\
\text { por fissionais de equipes da ESF } \\
\text { frente às demandas médico-sociais } \\
\text { apresentadas pelos usuários em } \\
\text { seu cotidiano de trabalho. }\end{array}$ & $\begin{array}{l}\text { O investimento no desenvolvimen- } \\
\text { to de competências, o fortaleci- } \\
\text { mento de estratégias de enfren- } \\
\text { tamento coletivas, assim como } \\
\text { maior articulação com as redes } \\
\text { de serviços e as lideranças locais, } \\
\text { mostram-se necessários para que } \\
\text { os profissionais de saúde atuem } \\
\text { com menor estresse, e assim con- } \\
\text { tribuam na consolidação da ESF. }\end{array}$ \\
\hline $\begin{array}{l}\text { A10- Batista, K.; Souto, BGA. } \\
\text { Percepções e práticas de agentes } \\
\text { comunitários de saúde na atenção } \\
\text { a usuários de drogas. ABCS health } \\
\text { science; v. } 42 \text {, n. } 3 \text {, p. } 129-136,2017 .\end{array}$ & LILACS & $\begin{array}{l}\text { Descrever as percepções e as } \\
\text { expectativas de um grupo de ACS } \\
\text { sobre usuários de álcool e outras } \\
\text { drogas (UAOD), sobre as quais } \\
\text { fundamentam suas respectivas } \\
\text { práticas de cuidado. }\end{array}$ & $\begin{array}{l}\text { Os ACS querem cuidar adequa- } \\
\text { damente dos usuários, mas não } \\
\text { sabem como fazer devido a que } \\
\text { possuem noções leigas sobre essas } \\
\text { pessoas e não recebem formação } \\
\text { técnica suficiente. }\end{array}$ \\
\hline
\end{tabular}

Fonte: construção dos autores

Quanto à caracterização dos estudos, evidenciou-se o predomínio de estudos realizados em Estratégias de Saúde da família (9) seguido de Unidade Básica de Saúde (1). Quanto aos participantes dos estudos, a maioria deles foi composta pelas equipes de saúde (8), seguido de enfermeiros (1) e Agentes Comunitários de Saúde (1).

Quanto aos anos de publicação dos estudos, 2014 foi o ano que mais se concentraram os estudos (3), seguido de 2018 (2). Nos anos de 2012, 2013, 2017, 2019 e 2020 foi realizado um estudo por ano. Quanto aos periódicos de publicação se destaca a Revista Saúde e Sociedade (2), seguida da ABCS health science, Texto Contexto em Enfermagem, Psicologia: Ciência e Profissão, Psicologia em Estudo, Saúde e Debate, Ciência e Saúde Coletiva, Revista da Escola de Enfermagem da Universdade de São Paulo e a Revista Brasilera de Enfermagem, com um estudo em cada revista. 
Após a análise dos dados evidenciou-se que um dos estudos busca identificar a incidência do uso de drogas na população e quais são as drogas mais consumidas, quais sejam o tabaco, hipnóticos, maconha, cocaína e crack (A1). Ainda, expõe o efeito positivo da Intervenção Breve (IB) na redução do consumo que causem risco às pessoas que fazem uso abusivo de drogas (PLATT et al., 2016; PATRÍCIO; FINNELL; BARROSO, 2016). Esse tipo de intervenção foi desenvolvido, especialmente, para pessoas com risco moderado relacionados ao uso de drogas, que pode evoluir para complicações tanto de saúde quanto sociais, legais, laborais ou econômicos. As IB podem ser realizadas por qualquer profissional de saúde capacitado. No entanto, a Atenção Básica é o local mais adequado para a aplicação de IB, a qual consiste em oferecer um breve conselho e que busque motivar as pessoas que fazem uso abusivo de drogas para uma mudança de hábitos (PEREIRA et al., 2013).

Além disso, evidenciou-se que o usuário de drogas não tem as suas demandas de saúde atendidas nos serviços de Atenção Básica, e por vezes, os profissionais de saúde não compreendem o uso de drogas como sendo uma necessidade de saúde (A2, A10) ou até mesmo não conhecem todas as pessoas que fazem uso abusivo de drogas do seu território de atuação (A3). Nesse sentido as práticas de saúde são insuficientes para essa população (A2). É possível identificar fragilidades da assistência à saúde dessas pessoas desde o momento em que ocorre a anamnese, haja vista que a maioria dos profissionais dificilmente questiona as pessoas se esses fazem uso ou não de drogas (A3).

Os enfermeiros, em especial aqueles que fazem parte dos cuidados de saúde primários ocupam uma importante posição na identificação e intervenção precoce junto às pessoas que apresentam consumo de risco ou nocivo referente ao álcool. Deste modo, enfatiza-se a necessidade de capacitação dos enfermeiros tanto para a utilização de instrumentos de triagem, como é o caso do Alcool Use Disorders Identification Test (AUDIT) como para a aplicação do protocolo de intervenção breve (JORGE; PEREIRA; BARROSO, 2017). É através dos instrumentos de triagem que o profissional de saúde conseguirá identificar as demandas reais das pessoas que fazem uso abusivo de drogas e assim construir uma linha de cuidados de acordo com suas necessidades.

Alguns estudos também mencionam as necessidades de melhorias para a assistência das pessoas que fazem uso abusivo de drogas, já que apontam para a necessidade de modificar o olhar sobre esses, a fim de evitar a segregação (A4) e o preconceito (A5, A6, A10). As pessoas que fazem uso abusivo de drogas são vistos como sendo aquelas pessoas com desvios de caráter. Essa afirmação é corroborada por um estudo realizado com gestantes usuárias de drogas nos serviços de saúde, a qual aponta a importância do cuidado em enfermagem livre de estigmas. A presença de estigma no cuidado à saúde cria barreiras significativas entre o profissional e o usuário, motivo esse que os distancia dos serviços de saúde, local que deveria ser de acolhimento ao invés de afastamento em decorrência de atitudes discriminatórias (VENTURA et al., 2020)

Também, os estudos desvelam desafios referentes ao desenvolvimento de um cuidado pautado no vínculo, na escuta e no acolhimento (A5) bem como, a necessidade de evitar a culpabilização do 
usuário pelo insucesso no seu tratamento (A7). Para tornar o cuidado em saúde mental humanizado é necessário promover a escuta pelo profissional de saúde, que exige uma posição livre de preconceitos socialmente construídos, para que ele possa perceber a pessoa assistida como um sujeito de direitos.

Ainda, é imprescindível compreender suas limitações para construir, no âmbito da Atenção Básica a humanização das práticas de promoção e prevenção, diagnóstico, tratamento e reabilitação das pessoas que fazem uso abusivo de drogas (SANTOS, 2019). Vivências revelam que quando os enfermeiros da Atenção Básica visualizam o usuário de drogas como problemático e de difícil adesão ao tratamento, eles fortalecem o um discurso social que cria obstáculo ao cuidado desse usuário e ainda instiga a busca por um culpado pelo insucesso do tratamento baseado na abstinência (QUEIROZ SUBRINHO et al., 2018).

Quanto as emergências que possam vir a ocorrer na ESF, os estudos mostram que há um número significativo de profissionais da saúde que não saberiam como abordar o usuário. Também, não possuem conhecimento da Rede de Atenção à Saúde (RAS) nessas situações de uso de drogas (A3, A8). Isso aponta para a carência que inicia desde a formação profissional (A7, A10).

De acordo com um estudo desenvolvido por Veríssimo et al. (2020), com uma equipe de enfermagem no contexto hospitalar, o cuidado ao usuário de drogas é considerado uma atividade frustrante e complexa. O estudo também traz uma lacuna no conhecimento da equipe acerca do cuidado e abordagem dessas pessoas. Assim, pode-se afirmar que a ausência de uma equipe interdisciplinar e as limitações na implementação de políticas públicas contribuem para a ausência de habilidades com essa população.

O investimento no desenvolvimento de competências dos profissionais, o fortalecimento de estratégias de enfrentamento coletivas, assim como uma maior articulação com as redes de serviços e as lideranças locais, mostram-se necessários para que os profissionais de saúde atuem com menor estresse, e assim contribuam na consolidação da ESF (A9). Estudo realizado com enfermeiras de UBS do estado do Espírito Santo revela estratégias que podem ser desenvolvidas nesses serviços com o intuito de modificar sentimento de impotência e, fortalecer vínculos entre enfermeiros e pessoas que fazem uso abusivo de drogas. Essas estratégias estão ligadas a criação ou manutenção de grupos de educação em saúde, fortalecimento do discurso da redução de danos por meio da capacitação da equipe, empoderamento das pessoas que fazem uso abusivo de drogas e o uso da consulta de enfermagem como um meio para a construção de vínculos e organização do cuidado a essa população (QUEIROZ SUBRINHO et al., 2018).

Diante do exposto, essa revisão mostra que ainda há muitos desafios no que tange à formação e capacitação dos profissionais de saúde para que os mesmos possam atender as pessoas que fazem uso abusivo de drogas na Atenção Básica com um cuidado que seja integral e humanizado bem como, romper com estigmas e preconceitos para uma melhor assistência. Em contrapartida, mostra que nos serviços onde há IB os resultados mostram-se satisfatórios. 


\section{CONCLUSÃO}

Através desse estudo de revisão que teve como objetivo identificar o que vem sendo produzido sobre a assistência à saúde das pessoas que fazem uso abusivo de drogas na ESF identificou-se que há um déficit significativo das equipes na construção do cuidado à essas pessoas, tanto no que envolve a identificação do uso de drogas quanto nas formas de intervir para um cuidado efetivo e humanizado. Ainda, desvela que é imprescindível capacitar as equipes de saúde para um atendimento humanizado e discutir questões relacionadas ao estigma, exclusão social e culpabilização.

É urgente a necessidade de envolver a equipe para a implicação sensível a esse cuidado, bem como na capacitação para a consolidação de um cuidado humanizado e que leve em consideração a singularidades das pessoas que fazem uso abusivo de drogas. Esse estudo vem contribuir para a mobilização frente à criação, manutenção e avaliação das Políticas Públicas de Saúde referente ao uso de drogas no País, principalmente vinculada aos processos de trabalho das equipes de saúde.

\section{AGRADECIMENTOS}

Agradecemos o apoio do Programa de Bolsas de Iniciação Científica à Pesquisa (PROBIC) da Universidade Franciscana (UFN) pelo incentivo à pesquisa.

\section{REFERÊNCIAS}

BRASIL. Ministério da Saúde. Reforma Psiquiátrica e política de saúde mental no Brasil. Brasília, DF, 2005.

BRASIL. Ministério da Saúde. Política Nacional de Atenção Básica. Brasília, DF. 2017.

BRASIL. Ministério da Saúde. Gabinete do Ministro. Portaria n 2.436, de 21 de setembro de 2017. Brasília, 2017

ESLABÃO, A. D. et al. Rede de cuidado em saúde mental: visão dos coordenadores da estratégia saúde da família. Revista Gaúcha de Enfermagem, v. 38, n. 1, e. e60973, 2017.

FARIAS, L. et al. Atitudes e práticas de profissionais atuantes na Estratégia Saúde da Família quanto à abordagem aos usuários de drogas no município de Campina Grande, Paraíba, Brasil. Ciência \& Saúde Coletiva, v. 24, n. 10, p. 3867-3878, 2019. 
FARIAS, L.M. da S. et al. O enfermeiro e a assistência a usuários de drogas em serviços de atenção básica. Revista de enfermagem UFPE on line, v. 11, n. 7, p. 1-10, 2017.

JORGE, F. M.; PEREIRA, M. O.; BARROSO, T. M. M. D. D. Intervenções breves na redução do consumo de álcool em utentes de uma unidade de saúde familiar. Revista de Enfermagem Referência, s/n, v. 14, p. 79-86, 2017.

LIRA, L. S. S.P. et al. Percepção da Equipe de Saúde da Família sobre o cuidado a usuários de drogas. Saúde em debate, Rio de Janeiro, v. 44, n. 125, p. 372-383, 2020.

PATRÍCIO, S. M.; FINNELL, D. S.; BARROSO, T. Efeito das intervenções breves na redução do consumo de álcool em indivíduos portadores do vírus da imunodeficiência humana. Revista de Enfermagem Referência, v. 4, n. 11, p. 41-49, 2016.

PEREIRA M.O. et al. Efetividade da intervenção breve para o uso abusivo de álcool na atenção primária: revisão sistemática. Revista Brasileira de Enfermagem, v. 66, n. 3, p. 420-28, 2013.

PLATT, L. et al. How effective are brief interventions in reducing alcohol consumption: Do the setting, practitioner group and content matter?: Findings from a systematic review and metaregression analysis. BMJ Open, v. 6, n. 8, e011473, 2016.

QUEIROZ SUBRINHO, L. et al. Cuidado ao consumidor de drogas: percepção de enfermeiros da Estratégia de Saúde da Família. Saúde e Sociedade, v. 27, n. 3, 834-844. 2018.

SALLUM, A. M. C, GARCIA, D. M, SANCHES, M. Dor aguda e crônica: revisão narrativa da literatura. Acta Paulista de Enfermagem, v. 25, n. 1, p. 150-4, 2012.

SANTOS, A. B. Escuta qualificada como ferramenta de humanização do cuidado em saúde mental na Atenção Básica. APS em Revista, v. 1, n. 2, p. 170-179, 2019.

SANTOS, F. F. D.; FERLA, A. A. Saúde mental e atenção básica no cuidado aos usuários de álcool e outras drogas. Interface-Comunicação, Saúde, Educação, v. 21, n. 63, p. 833-44, 2017.

SINIAK D.S. et al. Análise da rede social de um Centro de Atenção Psicossocial Atenção para álcool e outras drogas. Revista de Enfermagem da UFSM, v. 10 e32: 1-20. 
VENTURA, J., dos S. Silva. et al. Estigma associado a gestante/puérpera usuária de crack: ameaça que representa a instituição. Research, Society and Development, v. 9, n. 2, e. 122922083, 2020.

VERÍSSIMO, L. et al. O cuidar de usuários de álcool e outras drogas para equipe de enfermagem. Cadernos Brasileiros de Saúde Mental/Brazilian. Journal of Mental Health, v. 12, n. 32, p. 56-71, 2020. 